\title{
Malnutrition and body composition predict poor quality of life and reduced survival in ambulatory oncology patients receiving chemotherapy: a cross sectional study of 1015 patients
}

\author{
E.S. Sullivan ${ }^{1}$, E. Ní Bhuachalla ${ }^{1}$, S. Cushen ${ }^{1}$, D.G. Power ${ }^{2}$, A.M. Ryan ${ }^{1}$ and L. Daly ${ }^{1}$ \\ ${ }^{1}$ Nutritional Sciences, University College Cork, Cork, Ireland and \\ ${ }^{2}$ Medical Oncology, Mercy and Cork University Hospitals, Cork, Ireland.
}

Malnutrition is common in oncology and negatively impacts on clinical outcomes ${ }^{(1-4)}$. The aim of this study was to assess the nutritional status of a large cohort of Irish cancer patients and examine the impact of malnutrition on quality of life (QOL) and survival.

A cross sectional study of adult patients with gastrointestinal cancers undergoing chemotherapy between 2012-2016 was conducted. A survey was devised, incorporating clinical, nutritional, biochemical and QOL data (EORTC). Taste and smell alterations (TAs/SAs) were recorded. Nutritional status was evaluated using cancer cachexia (CC) diagnostic criteria and body composition was assessed using computed tomography images (at L3) using OsiriX software. Skeletal muscle index (cross sectional area/ height ${ }^{2}$, SMI) and mean muscle attenuation (MA) were obtained. Sarcopenia and low MA were defined using published cut points ${ }^{(1)}$. Univariate and multivariate analyses for overall survival were conducted using cox proportional hazards model; hazards ratios (HR) and corresponding $95 \%$ confidence intervals $(\mathrm{CI})$ were obtained.

1021 patients with solid tumours participated in the study, $56 \%$ were male with a median age of 64 years [IQR 55-71]. The majority of patients $(57 \%)$ were overweight or obese $\left(\right.$ BMI $\left.>25 \mathrm{~kg} / \mathrm{m}^{2}\right)$, while only $4 \%$ had a BMI $<18.5 \mathrm{~kg} / \mathrm{m}^{2}$. Colorectal cancer was the most prevalent $(27 \%)$, followed by gastro-oesophageal cancer (15\%). The majority (54\%) of patients had stage IV disease, $35 \%$ had lost $>5 \%$ body weight, $42 \%$ had CC, $39 \%$ were sarcopenic, $45 \%$ had low MA, $21 \%$ had both. In a subgroup of patients (n= 300$), 52 \%$ reported TAs, while $21 \%$ reported SAs while on chemotherapy. TAs were higher in those with appetite loss $(\mathrm{p}<0.001)$ and nausea and vomiting $(\mathrm{p}=0.014)$. Oral nutritional supplements (ONS) were prescribed to 74 patients $(25 \%), 53 \%$ of which did not enjoy taking them and $38 \%$ would never take ONS again. 95\% reported they would prefer to get calories from food as opposed to ONS. In terms of QoL, weight loss $>5 \%$ was significantly associated with a poorer global QoL score, and worse physical, role, emotional and social functional scores $($ all $\mathrm{p}<0.005)$ and higher symptoms such as fatigue, nausea and vomiting, pain, appetite and diarrhoea (all $\mathrm{p}<0.05$ ). WL > 5\%, CC, SMI and MA were associated with reduced survival, however, controlling for factors known to predict survival (age, gender, performance status, cancer type, stage and smoking status), only MA remained significant. Every 10-unit decrease in MA was associated with a $27 \%$ increase in mortality risk [HR 1.27 (95\% CI: 1.15-1.37), $p=0.001$ ]. SMI had no impact on survival.

Malnutrition and abnormal body composition features are common in patients receiving chemotherapy but are masked by excessive adiposity. WL adversely impacts on patient QoL. Low MA is a prognostic factor for OS in cancer patients receiving chemotherapy, whereas low SMI is not. More research is needed to determine the relevance of MA assessment in daily clinical practice. Food based strategies to enhance intake are preferred by cancer patients over ONS and more emphasis should therefore be placed on dietary counselling, high protein high calorie diets and exercise to improve patients' nutritional status and clinical outcomes.

1. Martin L, Birdsell L, MacDonald N et al. (2013) J Clin Oncol 31, 1539-1547.

2. Martin L, Senesse P, Gioulbasanis J et al. (2015) J Clin Oncol 33, 90-99.

3. Daly LE, Power DG, O'Reilly Á et al. (2017) Br J Cancer 116, 310-317.

4. Cushen SJ, Power DG, Teo MY et al. (2017) Am J Clin Oncol 40, 47-52. 